\title{
Preparation and Performance of Nano-hybrids Synthesized from Polyimide and Silica
}

\author{
S. M. M. Alam ${ }^{1}$ \\ College of Textile Technology, Tejgaon, Dhaka-1208, Bangladesh
}

Received 29 March 2009, accepted in revised form 24 November 2009

\begin{abstract}
Polyimide (PI)-inorganic like silica hybrid films were successfully prepared to combine the good performances of silica like tensile modulus, thermal stability etc. into organic PI. Polyamic acid (PAA), precursor of PI, was prepared from 3, 3', 4, 4'biphenyltetracarboxylic dianhydride (BPDA), p-phenylediamine (PDA) and in-situ formed silica was formed into PAA from tetraethoxysilane (TEOS) through in-situ sol-gel process. The films were transparent and became translucent in presence of up to $10 \%$ inorganic contents. The chemical structures were characterized by Fourier transform infrared spectroscopy (FTIR). The morphology of the films was investigated by scanning electronic microscopy (SEM). Differential scanning calorimetry (DSC), thermogravimetry analysis (TGA), stress-strain tests and dynamic mechanical analysis (DMA) were used to evaluate the performances of the films. The results indicated that the glass-transition temperatures $\left(T_{\mathrm{g}}\right)$ and decomposition temperatures of the PI-silica hybrid films were higher than those of pristine PI. Tensile modulus, tensile strength of PI increased prominently in presence of small amount (1\%) of silica in PI-silica hybrid.
\end{abstract}

Keywords: Polyimide; Hybrid; Sol-gel process.

C) 2010 JSR Publications. ISSN: 2070-0237 (Print); 2070-0245 (Online). All rights reserved.

DOI: $10.3329 /$ jsr.v2i1.2733 J. Sci. Res. 2 (1), 99-107 (2010)

\section{Introduction}

Aromatic PIs which are considered as super engineering materials due to their superior chemical resistance, high thermal stability and excellent mechanical properties, are used as insulating materials for electronics, high-performance gas-separation membrane, hightemperature adhesives and coatings, matrices for composites etc [1]. Even though PIs are thermally stable polymers, they can still degrade if exposed to a high temperature for a long period which leads to change in physical and/or chemical structure of the materials and affect their thermooxidative stability. Also good tensile properties is essential for PI which is used as alpha-ray shielding films, alignment films, interlayer insulation films, buffer coating films in electronic industries [2]. Moreover, most PIs exhibit relatively

${ }^{1}$ E-mail: dalim70@yahoo.com 
high co-efficient of thermal expansion (CTE) which limit their application for advanced electronic products [3]. In this situation, PI-silica hybrid prepared by in-situ sol-gel process will be an excellent approach to improve thermal stability, tensile modulus and CTE of PI due to high thermal stability and rigidity of silica. Till now, much research has carried out to develop the properties of PI [4-17]. Among the PIs, rigid PI from BPDA and PDA (Scheme 1) is a unique type of PI because of its high modulus, increased tensile modulus by cold drawing, remarkable spontaneous in-plane orientation, low water sorption, low stress relaxation co-efficient and minimum coefficient of thermal expansion [18-21]. So far, no research has been carried out to observe the thermal and mechanical properties of rigid PI from BPDA, PDA and silica hybrids through in-situ sol-gel process.

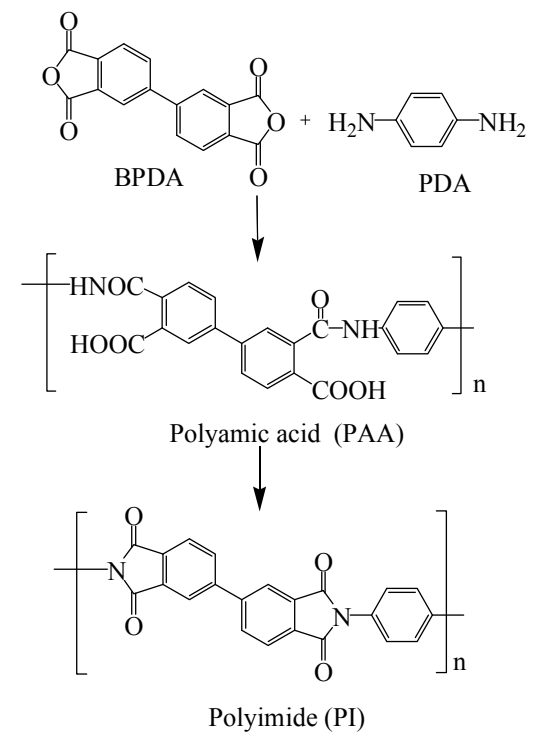

Scheme 1. Polyimide from BPDA and PDA.

In current research, effort was made to develop the tensile modulus, glass transition temperature, flame retardency property of rigid PI with different ratio of silica $(1,3,5$, $10 \%$ ) from tetraethoxysilane (TEOS) by sol-gel process. In-situ sol-gel process is a convenient technique to produce inorganic in presence of catalyst. In this research, no catalyst was added. It was expected that the carboxyl group of polyamic acid (PAA) would act as catalyst for sol-gel process. The silica ratio was limited to $10 \%$ due to decreased elongation and transparency of PI-hybrids. Optical transparency of PI films is of special importance for their use as flexible solar radiation protectors, orientation films in liquid crystal display devices, and optical half-wave plates for planar light wave circuits. After synthesized PI-silica hybrids, the thermo-mechanical performances and morphology of the hybrids were checked in details by using different apparatus and methods. 


\section{Experimental}

\subsection{Reagents}

TEOS from Wako Pure Chemical Industries Ltd., Japan was used as received. BPDA from UBE Industries Ltd., Japan and PDA from Tokyo Kasei Kogyo Co. Ltd, Japan were purified by sublimation. n-methyl-2-pyrrolidone (NMP) from Osaka Chemicals, Japan was dried by distillation under reduced pressure over sodium hydride. Hydrochloric acid (35\%) from Tokyo Kasei Kogyo Co. Ltd, Japan, was used as received. PI was prepared from BPDA and PDA in presence of NMP as solvent through polycondensation as shown in Scheme 1.

\subsection{Preparation of PI-silica hybrids by sol-gel process}

Into a flask equipped with mechanical stirrer, certain amount of PAA was stirred with required amount of TEOS for about half an hour. Water (double amount in mmol with respect of TEOS) was then added and stirred for $24 \mathrm{hrs}$. The solution was cast on glass plates by using doctor blade, dried at $60^{\circ} \mathrm{C}$ for $16 \mathrm{hrs}$, cured at $100^{\circ} \mathrm{C}, 200^{\circ} \mathrm{C}$ and $300^{\circ} \mathrm{C}$ for $1 \mathrm{~h}$ each to obtain hybrid films.

\subsection{Apparatus to measure performances of PI-silica hybrids}

Infra-red (IR) spectra were obtained with Jasco Spectrophotometer (Model FT/IR-420). Differential scanning calorimetry (DSC) was recorded using Rigaku Thermo Plus 2DSC8230 at a heating rate of $10^{\circ} \mathrm{C} / \mathrm{min}$ in nitrogen. Thermal gravimetric analysis (TGA) was performed with Rigaku Thermo Plus 2TG-DTA TG8120 at a heating rate of $5^{\circ} \mathrm{C} / \mathrm{min}$ in argon. Dynamic mechanical analysis (DMA) were conducted on Automatic Dynamic Viscoelastomer Rheovibron (Model ORIENTEC DDV-01FP) at $35 \mathrm{~Hz}$ at a heating rate of $4{ }^{\circ} \mathrm{C} / \mathrm{min}$. Tensile properties were recorded from at least 5 tests with Imada Seisaku-sho Model SV-3 at a cross-head speed of $1 \mathrm{~mm} / \mathrm{min}$ using films of $2 \mathrm{~cm}$ long. Scanning electron microscopy (SEM) studies were performed to check the particle size of the inorganics using a Hitachi S-4800 scanning electron microscope with the accelerating voltage of $10 \mathrm{kV}$ and emission current of $10 \mu \mathrm{A}$. The film surface were etched by aqueous $1 \mathrm{~N} \mathrm{NaOH}$ and then sputter-coated with Pt-Pd under an electric current of $15 \mathrm{~mA}$ at $6 \mathrm{~Pa}$ for 60s. Transparency was checked by JASCO V-550 UV/vis spectrophotometer in where all samples' thickness was about $0.03 \mathrm{~mm}$.

\subsection{Preparation of hybrid films}

Hybrid films were prepared through sol-gel process in to PAA. The sol-gel process consists of two steps--hydrolysis and condensation. In hydrolysis, silanol groups were formed from TEOS in presence of water and these silanols reacted with each other to 
form silica in condensation (scheme 2). The preparation of silica in PI was confirmed by FTIR (Fig. 1). In FTIR spectra, it was found that absorption band related to -Si-O-Si- at 1000 to $1100 \mathrm{~cm}^{-1}$ increased after increasing silica contents which proved the presence of silica in PI-silica hybrid after curing. The characteristic absorption peaks of imide groups at $1774 \mathrm{~cm}^{-1}$ ( $\mathrm{C}=\mathrm{O}$ symmetric stretching $), 1718 \mathrm{~cm}^{-1}(\mathrm{C}=\mathrm{O}$ asymmetric stretching) and $1361 \mathrm{~cm}^{-1}(\mathrm{C}=\mathrm{N}$ stretching) were observed after curing. Absorption stretches associated with aromatic $\mathrm{C}=\mathrm{C}$ bonds at $1517 \mathrm{~cm}^{-1}$ was also observed in the hybrid materials.

Imidization was also confirmed by DSC (Fig. 2). DSC was measured from the PIsilica hybrid films after $100^{\circ} \mathrm{C}$ curing. In DSC, endotherms happened due to imidization mainly. The amount of endotherm decreased with the increase of silica contents (Fig. 1) which proved the presence of silica in PI-silica hybrid.

\subsection{Morphological study}

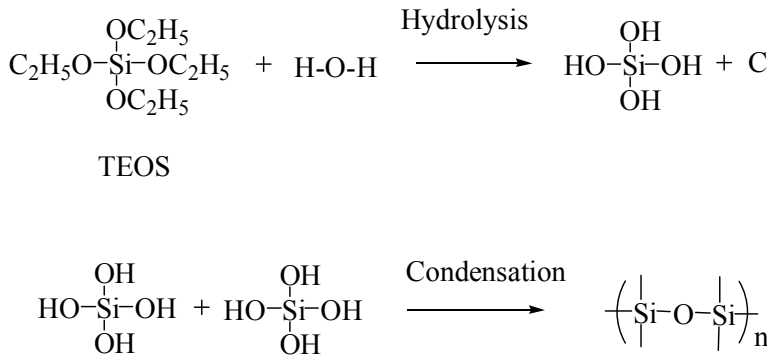

$$
\begin{aligned}
& \text { Silica }
\end{aligned}
$$

Scheme 2. Preparation of Silica.

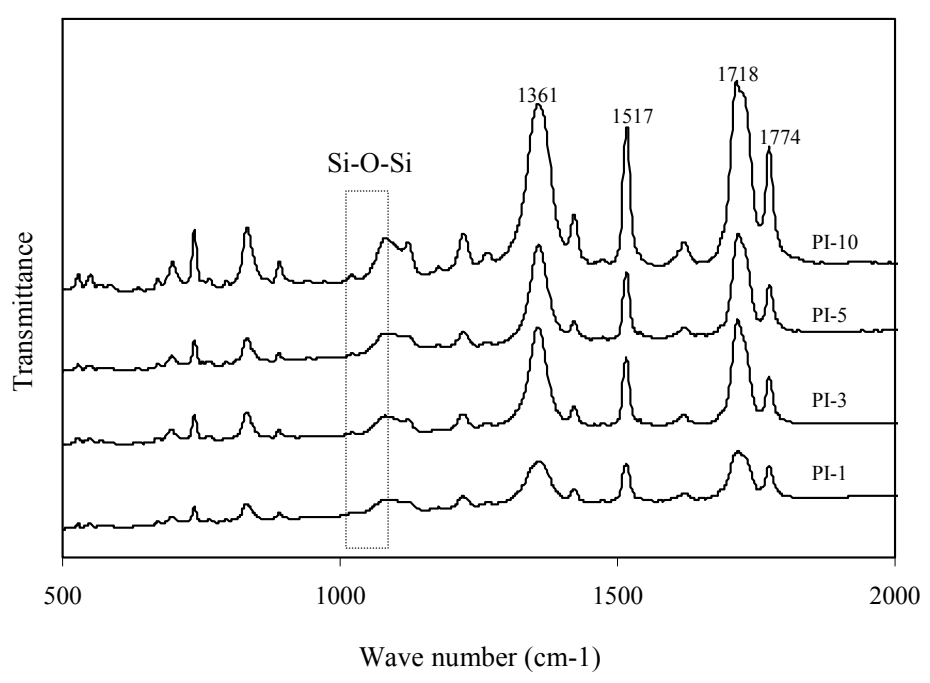

Fig. 1. IR-spectras of PI-silica hybrids 


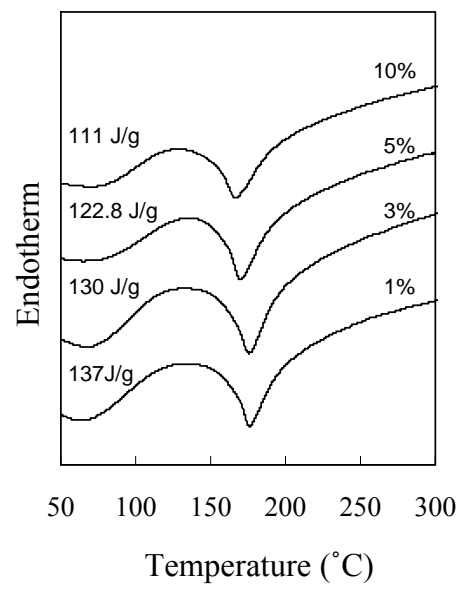

Fig. 2. DSC of PI-hybrids

Transparency of all hybrids were checked by UV-spectrophotometer. From UV-spectras, it was found that transparency became $76,73,59,50,27 \%$ at $700 \mathrm{~nm}$ wave no after adding 0, 1, 3, 5, 10\% silica (Fig. 3). Higher amount of inorganics related PI-silica hybrids became translucent which also proved the aggregation of inorganics at higher ratio. The size of silica particles in the hybrids were observed by SEM (Fig. 4). In case of lower silica $(1-5 \%)$ the particles were circular in shape and size about 30-60nm and the films were transparent. But the size of the particles became rectangular and larger (40-80nm) which also aggregated and made the film darker in case of PI-10 hybrid, and the aggregated film became darker in case of PI-10 hybrid.

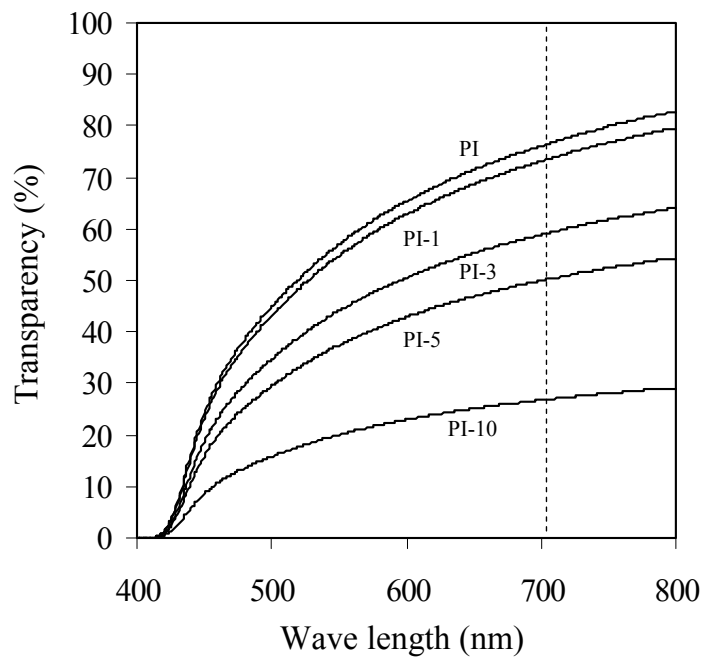

Fig. 3. Transparency of PI-hybrids. 

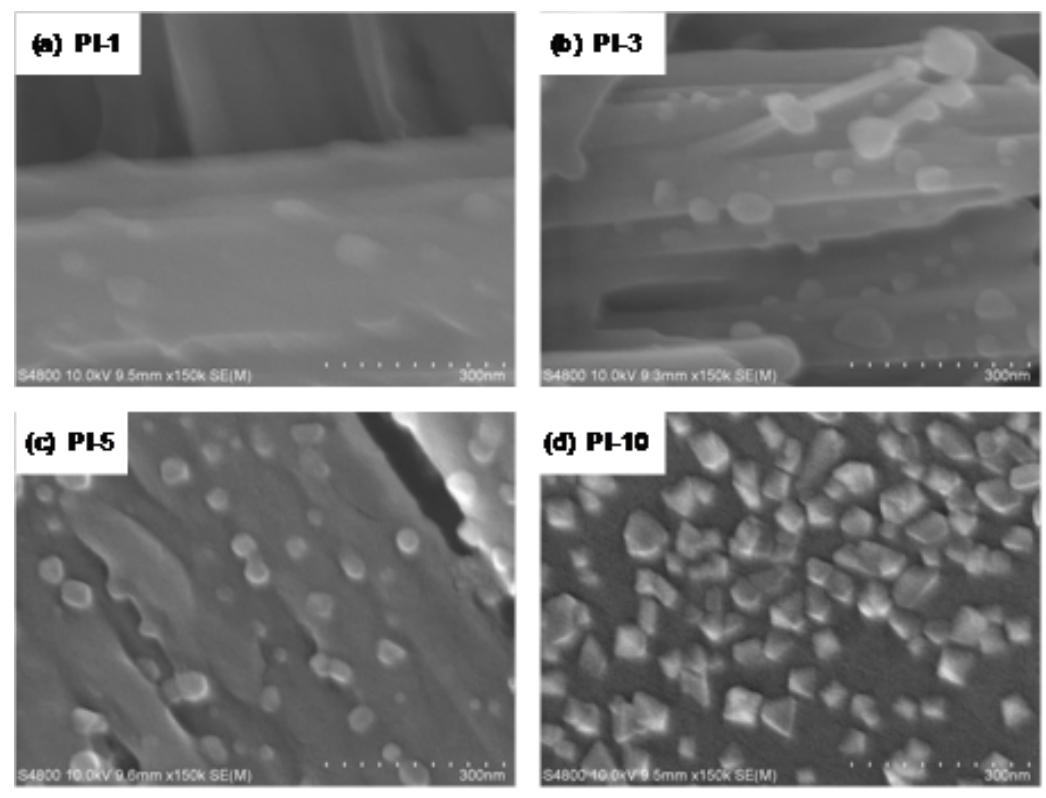

Fig. 4. SEM images of PI-hybrids.

\section{Results and Discussion}

\subsection{Tensile properties of PI-inorganics hybrids}

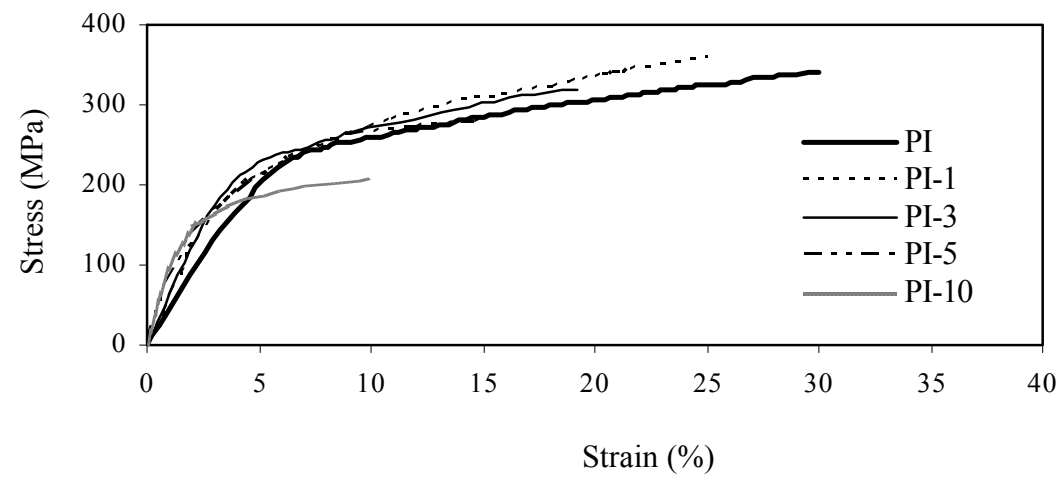

Fig. 5. Tensile properties of hybrids.

Inorganics have higher rigidity than organics but brittle behaviour which effect in hybrid properties. Pristine PI tensile modulus was 8.2 GPa which became 9.5, 10, 11, 11.5 GPa tensile modulus after inclusion 1 to $10 \%$ inorganics (Fig. 5). From IR-spectra, it was found that the higher contents of inorganic was present into hybrid after increasing silica ratio which also increased the modulus of PI-silica hybrids. Tensile strength increased 
from 340MPa to $360,300,280,210 \mathrm{MPa}$ in case of PI-silica (1 to $10 \%$ ) hybrids. Small amount of inorganic (1\%) increased the strength of organic-inorganic hybrid which was happened due to interfacial interaction. Silica decreased the elongation at break \% of PIsilica hybrids due to it's brittle behavior.

\subsection{Thermal properties of PI-inorganic nano- hybrids}

Thermal properties of PI-hybrids were investigated through DMA and TGA whose results are summarized in Table 1.

Table 1. Thermal properties of PI-silica hybrids.

\begin{tabular}{|c|c|c|c|c|c|}
\hline $\begin{array}{l}\text { Type } \\
\text { of } \\
\text { PI }\end{array}$ & $\begin{array}{c}\mathrm{T}_{\mathrm{g}}\left({ }^{\circ} \mathrm{C}\right)^{\mathrm{a}} \\
\text { from } \\
\mathrm{E}^{\prime \prime}\end{array}$ & $\begin{array}{c}\mathrm{T}_{\mathrm{g}}\left({ }^{\circ} \mathrm{C}\right)^{\mathrm{a}} \\
\text { from } \\
\tan \delta\end{array}$ & $\begin{array}{c}\mathrm{T}_{5} \\
\left({ }^{\circ} \mathrm{C}\right)^{\mathrm{b}}\end{array}$ & $\begin{array}{c}\mathrm{T}_{10} \\
\left({ }^{\circ} \mathrm{C}\right)^{\mathrm{b}}\end{array}$ & $\begin{array}{c}\text { Wt. residue } \\
(\%) \text { at } \\
800^{\circ} \mathrm{C}^{\mathrm{b}}\end{array}$ \\
\hline PI & 311 & 321 & 563 & 583 & 60.8 \\
\hline PI-1 & 337 & 361 & 572 & 584 & 62.3 \\
\hline PI-3 & 333 & 357 & 576 & 590 & 64.3 \\
\hline PI-5 & 330 & 351 & 571 & 592 & 65 \\
\hline PI-10 & 312 & 320 & 568 & 587 & 69.3 \\
\hline
\end{tabular}

a - measured by DMA.

b - measured by TGA under argon

\subsubsection{Dynamic mechanical analysis (DMA) of PI-inorganic nano- hybrids}

The viscoelastic properties of the hybrids were studied using DMA. The storage modulus $\left(E^{\prime}\right)$, loss modulus $\left(E^{\prime \prime}\right)$ and $\tan \delta$ were measured for $\mathrm{PI}_{-} \mathrm{SiO}_{2}$ hybrids. The increase of $T_{\mathrm{g}}$ of the hybrids in comparison with the neat PI can be attributed to maximizing the adhesion between the polymer and the inorganic surfaces because of the nanometer size which restricts the segmental motion near the organic and inorganic interface. Storage modulus of PI increased at $30^{\circ} \mathrm{C}$ after increasing the ratio of silica in case of PI-silica hybrids. Smaller size of inorganics have more reactive silanol group on the surface which made hbond with the carbonyl group of PI and provide higher interfacial interaction as well as higher $T_{\mathrm{g}}$ than the bigger particle size related hybrid.

\subsubsection{Thermal properties of PI-inorganic nano-hybrids}

TGA under argon (Fig. 6) were recorded for PI-inorganic hybrids to monitor the effect of silica on the thermal properties. PI-silica hybrids showed higher 5\% degradation temperature $\left(T_{5}\right), 10 \%$ degradation temperature $\left(T_{10}\right)$ and weight residue $\%$ at $800^{\circ} \mathrm{C}$ than pristine PI. In PI-silica hybrids, the incorporation of silica enhanced the thermal stability due to the higher thermal stability of silica. The weight residue $\%$ at $800^{\circ} \mathrm{C}$ of the PIsilica composites increased with increasing of silica ratio which shown flame retardant property of PI increased in presence of silica as well. 


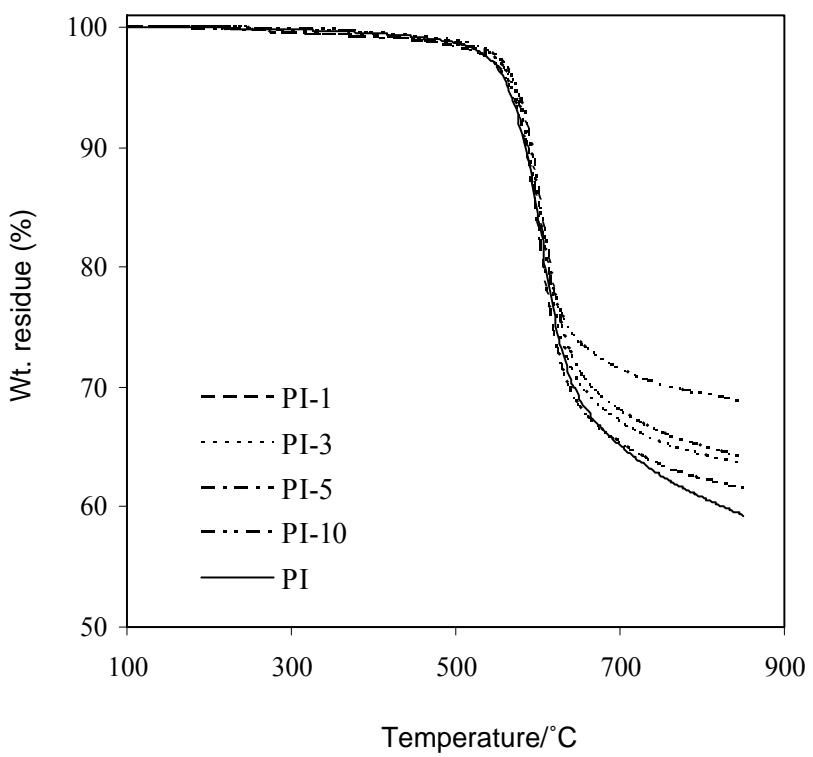

Fig. 6. TGA of PI-hybrids under argon.

\section{Conclusions}

The properties of inorganics are distributed nicely and prominently in rigid PI in case of rigid PI-silica hybrids. Higher thermal properties of inorganics increased the thermal behaviour of the hybrids. The rigidity of inorganics increased the tensile modulus of hybrids in presence of small amount of inorganics. $T_{\mathrm{g}}$ enhanced prominently in PIinorganic hybrids as well. PI-small amount of silica (1,3\%) showed better performance concerning modulus, strength, thermal stability than pristine PI.

\section{Referrences}

1. M. K. Ghosh and K. L. Mittal, Eds. (Marcel Dekker,New York,1996) p. 343.

2. Z. Martynenko and D. Soane, Polymers in microelectronics: Fundamentals and applications. (Elsevier, Amsterdam, 1989).

3. H. Kita, Y. Kusuki, K. Nakagawa, A. Nakamura,, K. I. Okamoto, N. Tanihara, H. Watanabe, and K. Tanaka, J. Polym. Sci Part B: Polym. Phys. 30, 1223, (1992). doi:10.1002/polb.1992.090301107

4. S. M. M. Alam, T. Agag,, and T. Takeichi, J. Photopolym Sci. \& Tech. 19(2), 293 (2006). doi:10.2494/photopolymer.19.293

5. S. M. M. Alam, T. Kawauchi, and T. Takeichi, J. Photopolym Sci. \& Tech. 20(2), 169 (2007). doi: $10.2494 /$ photopolymer.20.159

6. S. M. M. Alam, T. Kawauchi, and T. Takeichi, React. \& Funct. Polm. 67, 1218 (2007). doi:10.1016/j.reactfunctpolym.2007.07.003

7. T. Agag, T. Koga, and T. Takeichi, Polymer 42, 3399 (2001). doi:10.1016/S0032-3861(00)00824-7

8. J. C. Tang, G. L. Lin, H. C. Yang, G. J. Jiang, and Y. W. Chen-Yang, J. Appl. Polym. Sci. 104, 4096 (2007). doi:10.1002/app.26041 
9. M. Khalil, S. Saeed, and Z. Ahmad, J. Appl. Polym. Sci. 107, 1257 (2008). doi:10.1002/app. 27149

10. Sh. Al-Kandary, A. A. M. Ali, and Z. Ahmad, J. Appl. Polym. Sci. 98, 2521(2005). doi:10.1002/app.22233

11. L. Jiang, W. Wang, X. Wei, D. Wu, and R. Jin, J.Appl. Polym. Sci. 104, 1579 (2007). doi:10.1002/app. 25692

12. H. Wang, W. Zhong, P. Xu, and Q. Du, Macromol. Mater. Eng. 289, 793 (2004). doi:10.1002/mame.200400002

13. A. Al Arbash, Z. Ahmad, F. Al-Saghee, and A. A. M.Ali, J. Nanomaterials, Article ID.58648, 1 (2006). doi:10.1155/JNM/2006/58648

14. A. Morikawa, Y. Iyoku, M. Kakimoto, and Y. Imal, J Mater Chem. 2, 679 (1992). doi:10.1039/jm9920200679

15. H. Yi and G. Yi, J. Appl.. Polym. Sci. 88, 2210 (2003). doi:10.1002/app.11885

16. Q. Jiaqiang, Z. Hui, L. Xiangyang, Z. Xinyuan, and G. Yi, Polymer 48, 3379 (2007). doi:10.1016/j.polymer.2007.03.051

17. C. J. Cornelius and E. Marand, Polymer 43, 2385 (2002). doi:10.1016/S0032-3861(01)00803-5

18. M. Hasegawa, K. Okuda, M. Horimoto, Y. Shindo, R. Yokota, and M. Kochi, Macromolecule 30 (19), 5745 (1997). doi:10.1021/ma970551i

19. H. Han, J. Seo, M. Ree, S. M. Pyo, C. C. Gryte, Polymer 39 (13), 2963 (1998). ddoi:10.1016/S0032-3861(97)00602-2

20. H. Chung, C. Lee, and H. Han, Polymer 42, 319 (2001). doi:10.1016/S0032-3861(00)00341-4

21. S. T. Chen and H. H. Wagner, J. of Electronic Mat. 7, 797 (1993). doi:10.1007/BF02817357 